\section{Inquérito de saúde no Município de Campinas, São Paulo, Brasil (ISACamp): comparação de estimativas segundo posse de linha telefônica residencial}

\author{
Health survey in Campinas, São Paulo State, Brazil \\ (ISACamp): comparison of estimates according to \\ ownership of a residential telephone line
}

\author{
${ }^{1}$ Faculdade de Ciências \\ Médicas, Universidade \\ Estadual de Campinas, \\ Campinas, Brasil. \\ 2 Faculdade de Saúde \\ Pública, Universidade de \\ São Paulo, São Paulo, Brasil. \\ 3 Secretaria de Estado da \\ Saúde de São Paulo, \\ São Paulo, Brasil. \\ Correspondência \\ P. M. S. B. Francisco \\ Departamento de Medicina \\ Preventiva e Social, \\ Faculdade de Ciências \\ Médicas, Universidade \\ Estadual de Campinas. \\ Rua Tessália Vieira de \\ Camargo 126, Campinas, SP \\ 13084-971, Brasil. \\ primaria@fcm.unicamp.br
}

\begin{abstract}
The study assesses differences in socio-demographic, lifestyle, and health-related characteristics among adults with and without residential telephone lines using data from a health survey in Campinas, São Paulo State, Brazil, (2008-2009), through a population-based cross-sectional survey that included 2,637 adults (18 years and older). Descriptive statistics, chi-square tests, prevalence, and 95\% confidence intervals were used in the analysis. Estimates were also made of the bias associated with non-coverage of the population without telephones before and after adjusting for post-stratification. The impact of bias on the confidence intervals was assessed by the bias ratio. Some $76 \%$ of respondents owned residential telephone lines. Except for marital status, differences were observed in socio-demographic data according to ownership of residential telephones. After post-stratification adjustment, there was a decrease in bias estimates for variables associated with ownership of telephone lines. However, except for osteoporosis, post-stratification adjustment was insufficient to correct the non-coverage bias.
\end{abstract}

Health Surveys; Data Colection; Selection Bias
Priscila Maria Stolses Bergamo Francisco 1 Marilisa Berti de Azevedo Barros 1 Neuber José Segri 2 Chester Luiz Galvão Cesar 2 Maria Cecilia Goi Porto Alves 3

\section{Introdução}

Com a crescente realização de inquéritos populacionais nas últimas décadas no país, surgem questões metodológicas relevantes quanto ao delineamento amostral da pesquisa, procedimento de seleção das unidades (indivíduos) na coleta das informações, técnicas de análise da qualidade e validade dos dados obtidos, estratégias de pós-estratificação para controle da não resposta, avaliação do efeito de diferentes formas de obtenção das informações, tais como o modo de aplicação do instrumento de medida, tipo de informante, sequência das questões, tipo de vieses envolvidos 1,2, bem como a comparação de dados que sejam provenientes de diferentes modalidades de inquéritos ou de inquéritos desenvolvidos em períodos de tempo distintos 3 .

Entre as técnicas de coleta de dados, entrevistas face a face e via telefone vêm sendo amplamente utilizadas na realização de inquéritos de saúde conforme verificado em diversos estudos $4,5,6,7,8,9,10,11,12,13,14$

Nos inquéritos domiciliares, questões operacionais tais como localização de endereços sorteados e deslocamento em campo, muitas vezes dificultam a coleta de informações e, além disso, o tempo entre a realização das entrevistas e a disponibilização dos dados pode ser grande. Já os inquéritos via telefone possuem a capacidade de manter o controle de qualidade sobre todo o 
processo de coleta de dados e, além do menor custo, soma-se a agilidade do sistema em disponibilizar informações em tempo real 6,11,15,16,17,18, tornando esse método de coleta muito atraente para a realização de pesquisas nas diversas áreas. No entanto, apesar das vantagens, surge como principal limitação deste tipo de pesquisa a exclusão dos indivíduos residentes em domicílios que não possuem linha telefônica residencial $12,18,19,20$.

A não cobertura ocorre quando unidades na população de interesse não têm chance de ser selecionadas para a pesquisa 2 , podendo resultar em estimativas viciadas quando parte da amostra excluída (pessoas sem linha telefônica residencial) é diferente da parte que é observada (indivíduos que possuem linha de telefone residencial).

No que se refere às características demográficas e socioeconômicas, estilo de vida, estado de saúde e utilização de serviços de saúde, estudos apontam diferenças no perfil dos entrevistados segundo a presença de linha telefônica no domicílio 15,18,20,21,22.

Com o incremento da utilização de entrevistas via telefone residencial para obtenção de informações sobre saúde no país, surge ainda a necessidade de comparação de estimativas obtidas de inquéritos domiciliares e telefônicos, bem como a avaliação do impacto que a exclusão dos moradores sem linha telefônica residencial poderia ocasionar em tais estimativas 19,22. Quantificar a magnitude dessas diferenças é fundamental para que técnicas estatísticas de ajustes de pósestratificação apropriadas sejam propostas para reduzir os vícios decorrentes da não cobertura e, para tanto, torna-se necessário caracterizar o perfil dos entrevistados quanto à posse de linha telefônica residencial.

O objetivo do presente estudo foi avaliar diferenças quanto às características demográficas, socioeconômicas, comportamentos relacionados à saúde, estado de saúde, filiação a plano de saúde, uso de serviços e realização de exames preventivos, informações que foram obtidas de pessoas com e sem telefone residencial, utilizando os dados de inquérito domiciliar.

\section{Métodos}

Os dados utilizados neste estudo são provenientes do inquérito de saúde realizado no Município de Campinas (ISACamp), São Paulo, que é uma pesquisa de base populacional desenhada para analisar condições de vida, situação de saúde e uso de serviços de saúde, por meio de entrevistas domiciliares. A pesquisa foi realizada pelo Centro
Colaborador em Análise de Situação de Saúde do Departamento de Medicina Preventiva e Social da Faculdade de Ciências Médicas da Universidade Estadual de Campinas (UNICAMP).

A amostra do inquérito foi obtida por meio de amostragem probabilística, estratificada, por conglomerados e em dois estágios: setor censitário e domicílio. No primeiro estágio, 50 setores censitários foram sorteados com probabilidade proporcional ao tamanho (número de domicílios). O sorteio foi sistemático, ordenando-se de forma crescente os setores pelo percentual de chefes que possuíam nível universitário, produzindo uma estratificação implícita por escolaridade do chefe de família.

O número de pessoas para compor a amostra foi obtido tendo em conta a situação correspondente à máxima variabilidade para a frequência dos eventos estudados ( $p=0,50$ ), coeficiente de 95\% de confiança na determinação dos intervalos de confiança $(z=1,96)$, erro de amostragem entre 4 e 5 pontos percentuais e efeito de delineamento igual a 2, totalizando 1.000 indivíduos em cada domínio de idade, a saber: adolescentes (10 a 19 anos), adultos (20 a 59 anos) e idosos (60 anos e mais). Esperando-se uma taxa de $80 \%$ de resposta, o tamanho da amostra foi corrigido para 1.250. Para alcançar esse tamanho de amostra em cada domínio, após atualização em campo dos mapas dos setores sorteados e elaboração da listagem de endereços, foram selecionados, de forma independente, 2.150, 700 e 3.900 domicílios para adolescentes, adultos e idosos, respectivamente. Nesta pesquisa, em cada domicílio sorteado, foram entrevistados todos os moradores da faixa etária selecionada para aquele domicílio.

As informações foram adquiridas mediante questionário estruturado, previamente testado, e aplicado em entrevistas domiciliares, realizadas por entrevistadores treinados e supervisionados. As entrevistas foram digitadas em banco de dados criado no Epidata 3.1 (Epidata Association, Odense, Dinamarca). A integridade das entrevistas foi verificada em amostra aleatória de 5\% dos participantes por intermédio de reentrevista domiciliar ou telefônica, e não mostrou discrepância com os dados originais.

A variável dependente utilizada neste estudo foi a posse de linha telefônica residencial (sim ou não). Para a comparação das pessoas de 18 anos ou mais, segundo a posse de linha telefônica residencial, as seguintes variáveis independentes foram selecionadas:

Demográficas e socioeconômicas: sexo, idade, cor/raça autorreferida, situação conjugal, naturalidade, religião, escolaridade (em anos de estudo), renda per capita (em salários mínimos) e realização de atividade ocupacional remunerada 
(sim, não desempregado e outra, em que estão inseridos aposentados e pensionistas, donas de casa e estudantes entre outros).

Comportamentos relacionados à saúde: índice de massa corporal (IMC: $\mathrm{kg} / \mathrm{m}^{2}$ ), calculado com dados de peso e estatura referidos, tabagismo (não fumante, fumante e ex-fumante) e dependência de bebida alcoólica (AUDIT - Alcohol Use Disorders Identification Test) 23.

Condições de saúde e características relacionadas ao uso de serviços de saúde: autoavaliação da saúde, morbidade referida nos 15 dias anteriores à pesquisa, relato de hipertensão arterial, diabetes, osteoporose, asma/bronquite/ enfisema, procura por serviço ou profissional de saúde para problemas relacionados à saúde nos últimos 15 dias, internação hospitalar no último ano, consumo de medicamentos nos últimos três dias, consulta ao dentista nos últimos 12 meses, realização dos exames de mamografia entre as mulheres de 40 anos ou mais e de Papanicolaou nas de 20 anos ou mais e filiação a plano privado de saúde.

Para a comparação entre os grupos com e sem linha telefônica residencial, verificou-se a associação entre as variáveis selecionadas e a posse de linha residencial, por meio do teste quiquadrado com nível de 5\% de significância.

Na avaliação do efeito que a não cobertura da população sem linha telefônica residencial teria nas estimativas do inquérito, foram analisados, para as variáveis referentes aos comportamentos relacionados à saúde, condições de saúde e uso de serviços de saúde, associadas à posse de linha telefônica residencial, o vício associado à não cobertura e a razão de vício 24 .

As estimativas do vício absoluto foram obtidas pela expressão algébrica:

$$
\text { Vício }(P)=P_{t e l}-P \text {, }
$$

sendo $P_{t e l}$ : a estimativa da proporção da população com telefone residencial da amostra e $P$ : a estimativa da proporção da população total (com e sem telefone residencial). O efeito do vício nas inferências realizadas foi dimensionado pela razão de vício, por meio da expressão:

$$
R V(P)=\frac{\text { Vício }(P)}{\text { Erro Padrão }(P)}
$$

Na tabela sobre o efeito do vício no nível de confiança, criada por Cochran 25 (p. 14) para amostragem aleatória simples, estão indicados os níveis de confiança correspondentes a razões de vício de 0,02 a 1,50, que denotam alterações em relação ao nível de 95\% esperado para amostras não viciadas. Embora o autor sugira como regra prática que os vícios sejam considerados desprezíveis se os valores da razão de vício forem inferiores a 0,20 , trabalhos anteriores sobre vícios em pesquisas por telefone têm adotado o valor de 0,40 , como limite máximo aceitável para esse indicador 18,20. Valores superiores a 0,40 diminuem o nível previamente fixado de $95 \%$ dos intervalos de confiança para valores inferiores a 93,2\%.

Para verificar se os vícios diminuiriam após a utilização dos ajustes de pós-estratificação, foram obtidas novas estimativas das proporções, dos vícios e das razões de vício após a introdução desses ajustes que, por meio de pesos, promovem a equiparação da composição sociodemográfica da amostra àquela referente à população. Foi tomada como população (com e sem linha de telefone fixo) a amostra total do inquérito. Tais estimativas foram, então, comparadas às anteriores, possibilitando a avaliação do efeito da pós-estratificação.

No ajuste de pós-estratificação foram utilizadas as mesmas variáveis utilizadas pelo Sistema de Vigilância de Fatores de Risco e Proteção para Doenças Crônicas por Inquérito Telefônico (VIGITEL), considerando-se 36 estratos, a saber: sexo (masculino e feminino), faixa etária (18-24, 25-34, 35-44, 45-54, 55-64 e 65 e mais anos de idade) e nível de escolaridade (0-8, 9-11 anos e 12 ou mais anos de estudo) 11.

As análises dos dados foram realizadas pelo uso do programa Stata 11.0 (Stata Corp., College Station, Estados Unidos), cujos procedimentos para análise de inquéritos populacionais consideram aspectos do delineamento complexo da amostragem.

O projeto de pesquisa foi aprovado pela Comissão de Ética da UNICAMP (parecer $n^{\circ}$. 079/2007).

\section{Resultados}

Dos 2.637 indivíduos de 18 anos ou mais entrevistados, 2.634 responderam à pergunta sobre a posse de linha telefônica residencial no domicílio. Desses, 76,6\% (IC95\%: 70,9-82,2) referiram a posse de telefone fixo.

A média de idade dos adultos com linha de telefone $(\mathrm{n}=2.115)$ foi de 43,1 anos (IC95\%: 41,9$44,4)$, enquanto entre aqueles que não possuíam ( $n=519$ ) foi de 37,7 (IC95\%: 36,0-39,4). Na Tabela 1 , verifica-se, exceto para a situação conjugal ( $\mathrm{p}=0,10)$, associação estatisticamente significativa em relação a todas as variáveis sociodemográficas analisadas $(\mathrm{p}<0,05)$ entre as pessoas com e sem telefone. Entre as pessoas sem telefone predominam aquelas do sexo masculino, mais jovens, não brancos, que referiram estarem separadas ou divorciadas à época da pesquisa, 
Tabela 1

Características demográficas e socioeconômicas da população de 18 anos e mais, segundo presença de linha telefônica residencial no domicílio. ISACamp, Campinas, São Paulo, Brasil, 2008-2009.

\begin{tabular}{|c|c|c|c|c|c|c|c|}
\hline \multirow[t]{2}{*}{ Variável } & \multicolumn{2}{|c|}{ Com telefone } & \multicolumn{2}{|c|}{ Sem telefone } & \multicolumn{2}{|c|}{ Total } & \multirow[t]{2}{*}{ Valor de $p$ ** } \\
\hline & $n$ * & $\%$ & $n$ * & $\%$ & $n$ * & $\%$ & \\
\hline Sexo & & & & & & & $<0,001$ \\
\hline Masculino & 892 & 45,9 & 247 & 53,5 & 1.139 & 47,7 & \\
\hline Feminino & 1.223 & 54,1 & 272 & 46,5 & 1.495 & 52,3 & \\
\hline Faixa etária (anos) & & & & & & & $<0,001$ \\
\hline $18-24$ & 236 & 16,3 & 84 & 20,7 & 320 & 17,3 & \\
\hline $25-34$ & 187 & 21,1 & 85 & 31,0 & 272 & 23,4 & \\
\hline $35-44$ & 163 & 18,0 & 58 & 20,4 & 221 & 18,6 & \\
\hline $45-54$ & 166 & 18,1 & 40 & 14,0 & 206 & 17,2 & \\
\hline $55-64$ & 476 & 14,6 & 94 & 7,2 & 570 & 12,8 & \\
\hline 65 e mais & 887 & 11,9 & 158 & 6,7 & 1.045 & 10,7 & \\
\hline Cor/Raça & & & & & & & $<0,001$ \\
\hline Branca & 1.642 & 77,9 & 317 & 60,0 & 1.959 & 73,7 & \\
\hline Não branca & 470 & 22,1 & 201 & 40,0 & 671 & 26,3 & \\
\hline Situação conjugal & & & & & & & 0,100 \\
\hline Casado/Unido & 1.187 & 59,5 & 264 & 57,7 & 1.451 & 59,0 & \\
\hline Solteiro & 395 & 26,9 & 110 & 25,3 & 505 & 26,5 & \\
\hline Separado/Divorciado & 146 & 7,6 & 65 & 12,3 & 211 & 8,7 & \\
\hline Viúvo & 387 & 6,0 & 80 & 4,7 & 467 & 5,8 & \\
\hline Naturalidade & & & & & & & $<0,001$ \\
\hline Campinas & 676 & 41,2 & 137 & 32,0 & 813 & 39,0 & \\
\hline Outro município & 837 & 32,4 & 150 & 23,4 & 987 & 30,3 & \\
\hline Outro estado & 600 & 26,4 & 231 & 44,6 & 831 & 30,7 & \\
\hline Religião & & & & & & & 0,007 \\
\hline Católica & 1.296 & 55,4 & 256 & 41,1 & 1.552 & 52,0 & \\
\hline Evangélica & 531 & 28,5 & 168 & 37,1 & 699 & 30,5 & \\
\hline Outras/Sem religião & 283 & 16,1 & 95 & 21,8 & 378 & 17,5 & \\
\hline Escolaridade (anos) & & & & & & & $<0,001$ \\
\hline $0-8$ & 1.187 & 38,3 & 381 & 61,5 & 1.568 & 43,7 & \\
\hline $9-11$ & 428 & 27,7 & 98 & 27,9 & 526 & 27,8 & \\
\hline 12 e mais & 499 & 34,0 & 40 & 10,6 & 539 & 28,5 & \\
\hline 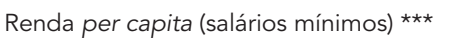 & & & & & & & $<0,001$ \\
\hline$\leq 1$ & 774 & 34,3 & 332 & 62,5 & 1.106 & 40,9 & \\
\hline$>1$ e $<3$ & 880 & 41,3 & 162 & 32,2 & 1.042 & 39,2 & \\
\hline$\geq 3$ & 461 & 24,4 & 25 & 5,3 & 486 & 19,9 & \\
\hline Atividade remunerada & & & & & & & $<0,001$ \\
\hline Trabalha & 846 & 63,3 & 243 & 66,8 & 1.089 & 10,9 & \\
\hline Desempregado & 54 & 4,4 & 42 & 9,3 & 96 & 5,6 & \\
\hline Outra & 1.214 & 32,3 & 234 & 23,9 & 1.448 & 30,3 & \\
\hline
\end{tabular}

* Número de indivíduos na amostra não ponderada;

** Valor de $p$ do teste qui-quadrado;

*** Salário mínimo vigente à época da pesquisa: janeiro a abril de 2008 (R\$ 415,00) de maio de 2008 a abril de 2009 (R\$ 450,00$)$.

migrantes de outros estados, não católicos, com menor nível educacional, menor renda e desempregadas, comparadas ao segmento com telefonia fixa.
No que se refere aos comportamentos relacionados à saúde, estado de saúde e utilização de serviços, diferenças significativas foram encontradas para filiação a plano médico de 
saúde, consumo de medicamentos nos três dias que antecederam a pesquisa, realização de consultas odontológicas nos últimos 12 meses, realização de mamografia entre as mulheres de 40 anos ou mais e presença de osteoporose autorreferida $(\mathrm{p}<0,05)$. Quanto à filiação a plano médico de saúde, os percentuais observados foram de $16,8 \%$ e $53,2 \%$ entre os que não possuíam e os que referiram posse de linha telefônica no domicílio, respectivamente (Tabela 2). Após ajuste por idade e sexo, as diferenças permaneceram para: consumo de medicamentos $(p=0,034)$, realização de consultas odontológicas ( $\mathrm{p}<0,001)$, filiação a plano médico de saúde $(\mathrm{p}<0,001) \mathrm{e}$ realização de mamografia $(p=0,008)$ entre as mulheres, ajustando-se apenas pela idade (dados não apresentados).

Ainda na Tabela 2, observam-se disparidades importantes quanto à realização de consultas odontológicas, já que apenas $34,6 \%$ dos entrevistados que não possuíam linha telefônica residencial consultaram o dentista nos 12 meses anteriores à pesquisa, enquanto para o grupo com telefone, esse percentual foi de cerca de $60 \%$.

A Tabela 3 apresenta os vícios das estimativas de prevalência possivelmente associados à não cobertura da população sem linha de telefone fixo, antes e depois do ajuste de pós-estratificação das variáveis associadas à posse do telefone. Observa-se que antes do ajuste todas as razões de vício estiveram acima de 0,40 , limite considerado aceitável para esse indicador. Após o ajuste, verificou-se redução do vício para todas as variáveis, no entanto, exceto para osteoporose autorreferida, as demais variáveis ainda apresentaram razões de vício entre 1,20 e 1,64 (consumo de medicamentos nos três dias que antecederam a pesquisa, realização de consultas odontológicas, realização de mamografia e filiação a plano médico de saúde).

Portanto, com os ajustes de pós-estratificação realizados, pôde-se verificar que houve redução do vício para todas as variáveis analisadas, mas apenas para a estimativa da prevalência de osteoporose, o nível de confiança manteve-se em patamar aceitável (superior a 93\%). A redução na razão de vício neste caso foi superior a $72 \%$. Em relação à filiação a plano médico de saúde, encontrou-se redução de $43,4 \%$; todavia, para esta e para as demais características associadas à posse de telefone residencial, as razões de vício denotaram um nível de confiança inferior a $80 \%$ para as estimativas por intervalo (Tabela 3) 25 .

\section{Discussão}

Neste estudo foi possível verificar diferenças entre indivíduos com e sem telefone em relação a todas as variáveis sociodemográficas analisadas, salvo para situação conjugal. Entre os indivíduos sem telefone, verificou-se maior porcentagem de pessoas do sexo masculino, mais jovens, não brancos ( $40 \%$ vs. $22,1 \%)$, migrantes de outros estados (cerca de $45 \%)$, não católicos $(58,9 \%$ vs. $44,6 \%$ ), com menor nível educacional (61,5\% com escolaridade inferior ou igual a 8 anos de estudo), menor renda $(62,5 \%$ com renda de até 1 salário mínimo) e desempregados (9,3\% vs. 4,4\%). Diferenças sociodemográficas entre os entrevistados, segundo posse de telefone fixo, também foram verificadas em outros estudos 15,18,20,22. Dados da Pesquisa Nacional por Amostra de Domicílios (PNAD) apontaram, na população adulta, maior chance de acesso a linhas telefônicas residenciais entre brancos e entre pessoas com maior escolaridade 18.

No que se refere aos comportamentos relacionados à saúde, condições de saúde e características relacionadas ao uso de serviços da população de 18 anos e mais, os dados analisados mostraram ainda que pessoas com telefone diferiram daquelas sem telefone em relação à osteoporose autorreferida, consumo de medicamentos nos três dias que antecederam a pesquisa, consultas odontológicas nos últimos 12 meses, realização de mamografia em mulheres com 40 anos ou mais e filiação a plano médico de saúde, com prevalências superiores para os indivíduos que referiram a posse de linha telefônica fixa no domicílio. Segri et al. 20 também verificaram diferenças em relação ao consumo de medicamentos, consultas odontológicas e realização de mamografia com dados de inquérito domiciliar realizado no município de São Paulo. O estudo não avaliou a associação entre filiação a plano médico de saúde e posse de telefone residencial, porém as variáveis relativas aos exames preventivos e consultas odontológicas foram analisadas também segundo tipo de serviço utilizado (SUS e não SUS), verificando-se associação estatística significativa entre o tipo de serviço e presença de linha telefônica residencial no domicílio 20 . Em estudo conduzido por Bernal \& Silva 18, a posse de pelo menos um plano de saúde esteve positivamente associada com o acesso à linha de telefone residencial. Quanto à osteoporose, as referências consultadas não consideraram esta condição entre as doenças crônicas avaliadas.

Não houve associação estatística entre posse de telefone fixo residencial e índice de massa corporal, morbidade referida, internação hospitalar nos últimos 12 meses, uso de serviços de saúde e 
Tabela 2

Comportamentos relacionados à saúde e estado de saúde da população de 18 anos e mais, segundo presença de linha telefônica residencial no domicílio. ISACamp, Campinas, São Paulo, Brasil, 2008-2009.

\begin{tabular}{|c|c|c|c|c|c|c|c|}
\hline \multirow[t]{2}{*}{ Variável } & \multicolumn{2}{|c|}{ Com telefone } & \multicolumn{2}{|c|}{ Sem telefone } & \multicolumn{2}{|c|}{ Total } & \multirow[t]{2}{*}{ Valor de $p$ ** } \\
\hline & $n$ * & $\%$ & $n$ * & $\%$ & $n$ * & $\%$ & \\
\hline $\mathrm{IMC}$ & & & & & & & 0,143 \\
\hline Normal & 978 & 50,0 & 292 & 55,2 & 1.270 & 51,2 & \\
\hline Sobrepeso & 318 & 31,8 & 148 & 30,9 & 881 & 31,6 & \\
\hline Obeso & 404 & 18,2 & 79 & 13,9 & 483 & 17,2 & \\
\hline Tabagismo & & & & & & & 0,399 \\
\hline Não fumante & 1.483 & 68,9 & 341 & 67,4 & 1.824 & 68,5 & \\
\hline Fumante & 287 & 18,0 & 96 & 21,5 & 383 & 18,8 & \\
\hline Ex-fumante & 343 & 13,1 & 81 & 11,1 & 424 & 12,7 & \\
\hline AUDIT & & & & & & & 0,317 \\
\hline Não dependente & 1.996 & 91,9 & 472 & 89,8 & 2.468 & 91,4 & \\
\hline Dependente & 117 & 8,1 & 44 & 10,2 & 161 & 8,6 & \\
\hline Autoavaliação da saúde & & & & & & & 0,230 \\
\hline Excelente/Muito boa & 709 & 42,0 & 136 & 34,5 & 845 & 40,2 & \\
\hline Boa & 1.212 & 50,7 & 324 & 58,4 & 1.536 & 52,5 & \\
\hline Ruim/Muito ruim & 194 & 7,3 & 59 & 7,1 & 253 & 7,3 & \\
\hline Morbidade referida & & & & & & & 0,173 \\
\hline Sim & 496 & 20,7 & 108 & 16,5 & 604 & 19,7 & \\
\hline Não & 1.618 & 79,3 & 410 & 83,5 & 2.028 & 80,3 & \\
\hline \multicolumn{8}{|c|}{ Relato das seguintes doenças/condições crônicas } \\
\hline Hipertensão & 796 & 20,7 & 164 & 16,8 & 960 & 19,8 & 0,079 \\
\hline Diabetes & 307 & 6,6 & 63 & 5,8 & 370 & 6,4 & 0,556 \\
\hline Osteoporose & 207 & 3,5 & 34 & 1,7 & 241 & 3,1 & 0,018 \\
\hline Asma/Bronquite/Enfisema & 109 & 4,1 & 27 & 3,5 & 136 & 3,9 & 0,598 \\
\hline Internação hospitalar & & & & & & & 0,595 \\
\hline $\operatorname{Sim}$ & 261 & 10,1 & 57 & 8,9 & 318 & 9,8 & \\
\hline Não & 1.854 & 89,9 & 462 & 91,1 & 2.316 & 90,2 & \\
\hline Uso de serviços de saúde & & & & & & & 0,558 \\
\hline Sim & 473 & 19,7 & 108 & 18,1 & 581 & 19,3 & \\
\hline Não & 1.642 & 80,3 & 411 & 81,9 & 2.053 & 80,7 & \\
\hline Consumo de medicamentos & & & & & & & 0,003 \\
\hline $\operatorname{Sim}$ & 1.493 & 59,2 & 292 & 47,3 & 1.785 & 56,5 & \\
\hline Não & 618 & 40,8 & 227 & 52,7 & 845 & 43,5 & \\
\hline Consultas odontológicas & & & & & & & $<0,001$ \\
\hline $\operatorname{Sim}$ & 1.007 & 59,7 & 152 & 34,6 & 1.159 & 53,8 & \\
\hline Não & 1.108 & 40,3 & 367 & 65,4 & 1.475 & 46,2 & \\
\hline \multicolumn{8}{|c|}{ 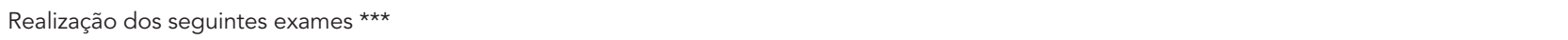 } \\
\hline Mamografia & 769 & 83,6 & 115 & 61,0 & 884 & 80,0 & $<0,001$ \\
\hline Papanicolau & 1.048 & 93,0 & 221 & 92,0 & 1.269 & 92,8 & 0,662 \\
\hline Plano de saúde & & & & & & & $<0,001$ \\
\hline Sim & 1.115 & 53,2 & 75 & 16,8 & 1.190 & 44,6 & \\
\hline Não & 998 & 46,8 & 443 & 83,2 & 1.441 & 55,4 & \\
\hline
\end{tabular}

AUDIT: Alcohol Use Disorders Identification Test.

* Número de indivíduos na amostra não ponderada;

** Valor de $\mathrm{p}$ do teste qui-quadrado;

*** Percentual das mulheres com 40 anos ou mais que disseram sim para mamografia e com 20 anos ou mais que disseram sim para o Papanicolaou. 
Estimativas de prevalências para pessoas de 18 anos ou mais com linha telefônica residencial e estimativas de vício, antes e após ajuste de pós-estratificação. ISACamp, Campinas, São Paulo, Brasil, 2008-2009.

\begin{tabular}{|c|c|c|c|c|c|c|c|c|}
\hline \multirow[t]{2}{*}{ Variável } & \multirow{2}{*}{$\begin{array}{l}\text { Resultado } \\
\text { do ISA- } \\
\text { Camp (\%) } \\
\text { * [1] }\end{array}$} & \multicolumn{3}{|c|}{ Antes da pós-estratificação } & \multicolumn{3}{|c|}{ Após a pós-estratificação ** } & \multirow{2}{*}{$\begin{array}{c}\text { Diminuição } \\
\text { da razão de } \\
\text { vício (\%) }\end{array}$} \\
\hline & & $\begin{array}{l}\text { Com tele- } \\
\text { fone (\%) * } \\
\text { antes do } \\
\text { ajuste [2] }\end{array}$ & $\begin{array}{c}\text { Vício antes } \\
\text { do ajuste } \\
{[2-1]}\end{array}$ & $\begin{array}{l}\text { Razão de } \\
\text { vício antes } \\
\text { do ajuste }\end{array}$ & $\begin{array}{l}\text { Com tele- } \\
\text { fone (\%) } \\
\text { * após do } \\
\text { ajuste [3] }\end{array}$ & $\begin{array}{c}\text { Vício após } \\
\text { o ajuste } \\
{[3-1]}\end{array}$ & $\begin{array}{c}\text { Razão de } \\
\text { vício após o } \\
\text { ajuste }\end{array}$ & \\
\hline Osteoporose autorreferida & 3,08 & 3,50 & 0,42 & 0,97 & 3,19 & 0,11 & 0,26 & 72,93 \\
\hline $\begin{array}{l}\text { Consumo de medicamentos (nos } 3 \text { dias que } \\
\text { antecederam a pesquisa) }\end{array}$ & 56,45 & 59,24 & 2,79 & 1,84 & 58,79 & 2,34 & 1,51 & 18,13 \\
\hline $\begin{array}{l}\text { Consultas odontológicas nos últimos } \\
12 \text { meses }\end{array}$ & 53,83 & 59,72 & 5,89 & 1,91 & 58,65 & 4,82 & 1,64 & 14,17 \\
\hline $\begin{array}{l}\text { Realização de mamografia (mulheres de } \\
40 \text { anos e mais) }\end{array}$ & 79,99 & 83,64 & 3,65 & 1,67 & 83,25 & 3,26 & 1,45 & 13,31 \\
\hline Filiação a plano médico de saúde & 44,65 & 53,18 & 8,53 & 2,13 & 49,42 & 4,77 & 1,20 & 43,37 \\
\hline
\end{tabular}

* Porcentagem na amostra ponderada;

** População total - estimativas baseadas apenas na informação do segmento com telefone.

realização de Papanicolaou, assim como verificado por Segri et al. 20. Em relação à percepção da própria saúde e ao tabagismo, também não foram encontradas diferenças significativas entre pessoas segundo posse de telefone residencial, diferentemente do que foi observado em outros estudos 20,22. As diferenças encontradas podem ser casuais ou decorrentes de perfis diferenciados das populações investigadas em relação às características investigadas, revelando a necessidade de outros estudos para compreender como essas diferenças se comportam em situações distintas. Para o autorrelato de diabetes, os achados deste estudo concordam com Ford 22, Bernal 26 e Segri et al. 20 que também não encontraram diferenças segundo posse de telefone residencial.

Para as variáveis analisadas neste estudo que apresentaram associação com a posse de telefone fixo, a maior estimativa de vício antes do ajuste de pós-estratificação foi de $8,5 \%$ para a proporção de pessoas que referiram filiação a plano médico de saúde. Após o ajuste, houve redução do vício para todas as variáveis analisadas e os valores foram inferiores a 5\%. Segundo Anderson et al. 27, embora as pessoas com telefone sejam diferentes daquelas sem telefone em relação a diversas características, estudos epidemiológicos utilizando entrevistas por telefone de amostras representativas da população podem não ser tão afetadas pelo viés de cobertura telefônica, desde que ela seja suficientemente elevada. Contudo, este estudo verificou que, apesar de a taxa de cobertura de linhas telefônicas residenciais em Campinas ser superior a $70 \%$, valor assinalado por Bernal \& Silva 18 como necessário para a realização de inquéritos epidemiológicos telefônicos, a exclusão de pessoas sem linha de telefone residencial resultou em estimativas viciadas, com alterações importantes do nível de confiança pré-fixado para os intervalos de confiança ${ }^{25}$. As razões de vício, que permitem dimensionar alterações potenciais do nível de confiança dos intervalos de confiança, provocadas pela presença do vício foram todas superiores ao nível máximo tolerado de 0,40 .

Particularmente no que se refere à realização de mamografia, a magnitude das diferenças entre as coberturas verificadas com dados da PNAD2003 para mulheres residentes em domicílios com e sem telefone fixo, evidenciou que dados referentes apenas à população com telefone fixo podem representar um viés de seleção, principalmente em municípios com baixa cobertura por telefonia fixa ${ }^{12}$. Neste caso, para corrigir esse viés, o ajuste de pós-estratificação poderia ser utilizado 28,29, mas os achados deste estudo corroboram os achados de Segri et al. 20, apontando que, mesmo após o ajuste de pós-estratificação, a estimativa da cobertura de mamografia apresenta vícios não desprezíveis.

Para as variáveis analisadas neste estudo, o principal resultado foi verificar que, exceto para osteoporose, o ajuste de pós-estratificação foi insuficiente para corrigir o vício de não cobertura, ou seja, para as estimativas da prevalência de consumo de medicamentos nos três dias que an- 
tecederam à pesquisa, consultas odontológicas nos últimos 12 meses, realização de mamografia em mulheres com 40 anos ou mais e filiação a plano médico de saúde, mesmo com o ajuste realizado, o vício continuou comprometendo as estimativas, caso elas fossem calculadas com uso apenas da parcela da população que possui telefone residencial.

Inquéritos telefônicos como o Behavioral Risk Factor Surveillance System (BRFSS) nos Estados Unidos e o VIGITEL, no Brasil, utilizam procedimentos de amostragem complexa e ajustes de pós-estratificação para minimizar o vício decorrente da ausência de dados da população sem telefone residencial. O inquérito americano considera no ajuste de pós-estratificação, o sexo, a idade e a variável raça/cor ao invés da escolaridade utilizada pelo VIGITEL 11,30.

Neste estudo, o ajuste de pós-estratificação, que levou em conta as mesmas variáveis utilizadas pelo VIGITEL (sexo, idade e escolaridade), reduziu o vício de todas as variáveis estudadas, mas quatro das cinco variáveis avaliadas ainda continuaram viciadas. Os resultados revelam que os ajustes de pós-estratificação são essenciais para corrigir as estimativas obtidas apenas com dados da população que possui telefone residencial, e aponta para a necessidade de novos estudos que avaliem se, além das variáveis sociodemográficas comumente utilizadas nos ajustes, a incorporação de outras variáveis pode minimizar os vieses associados às estimativas.

Estudos que utilizem amostras em que nem todas as unidades na população alvo têm chance de ser selecionadas para a pesquisa, como os inquéritos via telefone, devem considerar os vícios potenciais implícitos em cada variável estudada, já que "o grau do vício em pesquisas que excluem domicílios sem linha telefônica é um função de dois fatores: a magnitude da diferença entre aqueles que possuem e não possuem telefones em termos da variável que está sendo medida e a magnitude da porcentagem de domicílios sem telefone" 19,27.

Este estudo buscou quantificar as diferenças nas estimativas de prevalência para algumas variáveis que vêm sendo coletadas por meio de inquéritos domiciliares e telefônicos realizados na área da saúde e apresentou resultados complementares à literatura nacional sobre o tema. A utilização de técnicas estatísticas de ajustes de pós-estratificação buscou avaliar, para essas variáveis, a redução dos vícios decorrentes da não cobertura. Embora as diferenças relativas encontradas sejam grandes, as diferenças absolutas foram inferiores a 5 pontos percentuais. Estudos que incluam outras variáveis medidas em inquéritos domiciliares de saúde devem ser realizados, tendo em conta também os diferentes níveis de cobertura, para validar as informações obtidas por inquéritos telefônicos, que constituem importante modalidade de pesquisa e se destacam como ferramenta poderosa na vigilância de fatores de risco, fornecendo subsídios para o incremento de políticas voltadas à promoção da saúde no país.

\section{Conclusões}

Este estudo evidenciou que a utilização de técnicas de ajuste de pós-estratificação pode reduzir os vícios da não cobertura em pesquisas realizadas por telefone. Entretanto, deve-se considerar que, mesmo após o ajuste de pós-estratificação, a exclusão dos moradores sem linha telefônica residencial pode comprometer as inferências realizadas, a depender da variável investigada. 


\section{Resumo}

O estudo avalia diferenças quanto às características sociodemográficas, de estilo de vida e de condições de saúde entre adultos com e sem linha telefônica residencial, valendo-se de dados de inquérito domiciliar de saúde realizado em Campinas, São Paulo, Brasil (2008/2009). Trata-se de estudo transversal de base populacional com 2.637 adultos (18 anos e mais). Análises descritivas, testes qui-quadrado, prevalências e respectivos intervalos de 95\% de confiança foram calculados. Estimaram-se os vícios associados à não cobertura da população sem telefone antes e após o ajuste de pós-estratificação. O impacto do vício nos intervalos de confiança foi avaliado pela razão de vício. Cerca de $76 \%$ dos entrevistados possuíam linha telefônica residencial. Exceto para situação conjugal, foram observadas diferenças sociodemográficas segundo posse de telefone. Após o ajuste de pós-estratificação, houve redução do vício das estimativas para as variáveis associadas à posse de linha telefônica, no entanto, exceto para osteoporose, o ajuste de pós-estratificação foi insuficiente para corrigir o vício de não cobertura.

Inquéritos Epidemiológicos; Coleta de Dados; Viés de Seleção

\section{Colaboradores}

P. M. S. B. Francisco elaborou a proposta do artigo, planejou, programou e executou as análises estatísticas, realizou a revisão da literatura e redigiu o artigo. N. J. Segri contribuiu na elaboração da proposta, planejamento e execução das análises estatísticas, realizou a revisão da literatura e redação do artigo. M. B. A. Barros elaborou a proposta do artigo, revisou a análise e interpretação dos dados e contribuiu na revisão crítica e na redação do texto. C. L. G. Cesar participou da elaboração da proposta do artigo e contribuiu na revisão crítica do texto. M. C. G. P. Alves revisou as análises estatísticas e as interpretação dos dados, contribuindo também na revisão crítica do texto.

\section{Agradecimentos}

À Fundação de Amparo à Pesquisa do Estado de São Paulo (FAPESP) pelas bolsas de pós-doutorado de P. M. S. B. Francisco e de doutorado de N. J. Segri e ao Conselho Nacional de Desenvolvimento Científico e Tecnológico (CNPq) pela bolsa de produtividade de M. B. A. Barros e C. L. G. Cesar.

\section{Referências}

1. Kasprzyk D. Measurement error in household surveys: sources and measurement. In: Statistics Division, Department of Economic and Social Affairs, United Nations, editors. Household sample surveys in developing and transition countries. New York: United Nations; 2005. p. 171-98. (Series F, 96).

2. Lepkowski J. Non-observation error in household surveys in developing countries. In: Statistics Division, Department of Economic and Social Affairs, United Nations, editors. Household sample surveys in developing and transition countries. New York: United Nations; 2005. p. 149-69. (Series F, 96).

3. Lee S, Davis WW, Nguyen HA, McNeel TS, Brick JM, Flores-Cervantes I. Examining trends and averages using combined cross-sectional survey data from multiple years (CHIS Methodology Paper). http:// www.chis.ucla.edu/pdf/paper_trends_averages.pdf (acessado em 02/Jun/2010).
4. Lima-Costa MF, Barreto SM, Giatti L. Condições de saúde, capacidade funcional, uso de serviços de saúde e gastos com medicamentos da população idosa brasileira: um estudo descritivo baseado na Pesquisa Nacional por Amostra de Domicílios. Cad Saúde Pública 2003;19:735-43.

5. Galán I, Rodríguez-Artalejo F, Tobias A, Gandarillas A, Zorrilla B. Vigilancia de los factores de riesgo de las enfermedades no transmisibles mediante encuesta telefónica: resultados de la Comunidad de Madrid en el período 1995-2003. Gac Sanit 2005; 19:193-205.

6. Monteiro CA, Moura EC, Jaime PC, Lucca A, Florindo AA, Figueiredo ICR, et al. Monitoramento de fatores de risco para as doenças crônicas por entrevistas telefônicas. Rev Saúde Pública 2005; 39:47-57. 
7. Chiu TTW, Leung ASL. Neck pain in Hong Kong: a telephone survey on prevalence, consequences, and risk groups. Spine 2006; 31:540-4.

8. Bernards S, Graham K, Demers A, Kairouz S, Wells S. Gender and the assessment of at-risk drinking: evidence from the GENACIS Canada (2004-2005) telephone survey version of the AUDIT. Drug Alcohol Depend 2007; 88:282-90.

9. Marks GB, Abramson MJ, Jenkins CR, Kenny P, Mellis CM, Ruffin RE, et al. Asthma management and outcomes in Australia: a nation-wide telephone interview survey. Respirology 2007; 12:212-9.

10. Fahimi M, Link M, Mokdad A, Schwartz DA, Levy P. Tracking chronic disease and risk behavior prevalence as survey participation declines: statistics from the Behavioral Risk Factor Surveillance System and other national surveys. Prev Chronic Dis 2008; 5:A80.

11. Moura EC, Morais Neto OL, Malta DC, Moura L, Silva NN, Bernal R, et al. Vigilância de Fatores de Risco para Doenças Crônicas por Inquérito Telefônico nas capitais dos 26 estados brasileiros e no Distrito Federal (2006). Rev Bras Epidemiol 2008; 11 Suppl 1:20-37.

12. Viacava F, Souza-Junior PRB, Moreira RS. Estimativas da cobertura de mamografia segundo inquéritos de saúde no Brasil. Rev Saúde Pública 2009; 43 Suppl 2:117-25.

13. Barreto SM, Figueiredo RC. Doença crônica, auto-avaliação de saúde e comportamento de risco: diferença de gênero. Rev Saúde Pública 2009; 43 Suppl 2:38-47.

14. Francisco PMSB, Belon AP, Barros MBA, Carandina L, Alves MCGP, Goldbaum M, et al. Diabetes autoreferido em idosos: prevalência, fatores associados e práticas de controle. Cad Saúde Pública 2010; 26:175-84.

15. Marcus AC, Crane LA. Telephone surveys in public health research. Med Care 1986; 24:97-112.

16. Lavrakas PJ. Telephone survey methods: sampling, selection and supervision. London: Sage Publications; 1990.

17. Nelson DE, Powell-Griner E, Town M, Kovar MG. A comparison of national estimates from the National Health Interview Survey and the Behavioral Risk Factor Surveillance System. Am J Public Health 2003; 93:1335-41.

18. Bernal R, Silva NN. Cobertura de linhas telefônicas residenciais e vícios potenciais em estudos epidemiológicos. Rev Saúde Pública 2009; 43:421-6.
19. Kempf AM, Remington PL. New challenges for telephone survey research in the twenty-first century. Annu Rev Public Health 2007; 28:113-26.

20. Segri NJ, Cesar CLG, Barros MBA, Alves MCGP, Carandina L, Goldbaum M. Inquérito de saúde: comparação dos entrevistados segundo posse de linha telefônica residencial. Rev Saúde Pública 2010; 44:503-12.

21. Weeks MF, Kulka RA, Lessler JT, Whitmore RW. Personal versus telephone surveys for collecting household health data at the local level. Am J Public Health 1983; 73:1389-94.

22. Ford ES. Characteristics of survey participants with and without a telephone: findings from the Third National Health and Nutrition Examination Survey. J Clin Epidemiol 1998; 51:55-60.

23. Barbor TE, La Fuente JR, Saunders J, Grant M. AUDIT - the alcohol use disorders identification test: guidelines for use in primary health care. Geneva: World Health Organization; 1992.

24. Kish L. Survey sampling. New York: John Wiley; 1965.

25. Cochran WG. Sample techniques. New York: John Wills \& Sons; 1977.

26. Bernal R. Linhas telefônicas residenciais: usos em inquéritos epidemiológicos no Brasil [Tese de Doutorado]. São Paulo: Faculdade de Saúde Pública, Universidade de São Paulo; 2006.

27. Anderson JE, Nelson DE, Wilson, RW. Telephone coverage and measurement of health risk indicators: data from the National Health Interview Survey. Am J Public Health 1998; 88:1392-5.

28. Centers for Disease Control and Prevention. Behavioral Risk Factor Surveillance System - BRFSS: operational and user's guide. Atlanta: Centers for Disease Control and Prevention; 2006.

29. Secretaria de Vigilância em Saúde, Ministério da Saúde. VIGITEL Brasil 2008. Vigilância de fatores de risco e proteção para doenças crônicas por inquérito telefônico. Brasília: Ministério da Saúde; 2009.

30. Centers for Disease Control and Prevention. Technical documents and survey data. Behavioral Risk Factor Surveillance System - BRFSS weighting formula. http://www.cdc.gov/brfss/technical_info data/weighting.htm (acessado em 31/Mai/2011).

Recebido em 03/Mar/2011

Versão final reapresentada em 22/Jun/2011

Aprovado em 27/Jun/2011 